\title{
Measuring Interlayer Shear Stress in Bilayer Graphene
}

\author{
Guorui Wang, ${ }^{1,5}$ Zhaohe Dai, ${ }^{1,3,4}$ Yanlei Wang, ${ }^{2}$ PingHeng Tan, ${ }^{6}$ Luqi Liu, ${ }^{1, *}$ Zhiping Xu, ${ }^{2, \dagger}$ \\ Yueguang Wei, ${ }^{3}$ Rui Huang, ${ }^{4}$ and Zhong Zhang ${ }^{1, \$}$ \\ ${ }^{1}$ CAS Key Laboratory of Nanosystem and Hierarchical Fabrication, CAS Center for Excellence in Nanoscience, \\ National Center for Nanoscience and Technology, Beijing 100190, China \\ ${ }^{2}$ Applied Mechanics Laboratory, Department of Engineering Mechanics and Center for Nano and Micro Mechanics, \\ Tsinghua University, Beijing 100084, China \\ ${ }^{3}$ State Key Laboratory of Nonlinear Mechanics, Institute of Mechanics, Chinese Academy of Sciences, Beijing 100190, China \\ ${ }^{4}$ Center for Mechanics of Solids, Structures and Materials, Department of Aerospace Engineering and Engineering Mechanics, \\ The University of Texas at Austin, Austin, Texas 78712, USA \\ ${ }^{5}$ CAS Key Laboratory of Mechanical Behavior and Design of Materials, Department of Modern Mechanics, \\ University of Science and Technology of China, Hefei 230027, China \\ ${ }^{6}$ State Key Laboratory of Superlattices and Microstructures, Institute of Semiconductors, \\ Chinese Academy of Sciences, Beijing 100083, China
}

(Received 15 April 2017; published 17 July 2017)

\begin{abstract}
Monolayer two-dimensional (2D) crystals exhibit a host of intriguing properties, but the most exciting applications may come from stacking them into multilayer structures. Interlayer and interfacial shear interactions could play a crucial role in the performance and reliability of these applications, but little is known about the key parameters controlling shear deformation across the layers and interfaces between 2D materials. Herein, we report the first measurement of the interlayer shear stress of bilayer graphene based on pressurized microscale bubble loading devices. We demonstrate continuous growth of an interlayer shear zone outside the bubble edge and extract an interlayer shear stress of $40 \mathrm{kPa}$ based on a membrane analysis for bilayer graphene bubbles. Meanwhile, a much higher interfacial shear stress of 1.64 MPa was determined for monolayer graphene on a silicon oxide substrate. Our results not only provide insights into the interfacial shear responses of the thinnest structures possible, but also establish an experimental method for characterizing the fundamental interlayer shear properties of the emerging 2D materials for potential applications in multilayer systems.
\end{abstract}

DOI: 10.1103/PhysRevLett.119.036101

Two-dimensional (2D) materials, with monolayer crystalline structures and unusual physical characteristics, have been fueling extensive research on their use in semiconductors, electronics, batteries, and composites [1]. For most exciting applications of $2 \mathrm{D}$ crystals, the emergence of multilayer structures is inevitable because of the production process limitations [2,3] and, more importantly, the functional and operational requirements [4-7]. Indeed, ordered structures comprised of two or more layers represent a wide class of materials, and their extraordinary properties can be modulated by taking advantage of interlayer interactions. For instance, layered stacking of different 2D materials, generally called van der Waals (vdW) heterostructures, exhibit exceptional electronic and photonic properties [8]. From a classic view of multilayer systems, one of the most critical issues that determine its overall performance, durability, and even fabrication process lies in the interfacial load transfer [9]. For the atomically thin layers, the interfacial effects could be even more critical because fundamentally thermal, electronic, optical, and tribological properties could all be affected by the interlayer deformation (especially under shear mode) of multilayer structures [10-12], whereas corresponding mechanical parameters are far from well characterized.

Significant developments have been achieved in recent years in characterizing mechanical properties of graphene and other 2D crystals. The multilayer system is typically treated as a single sheet where the interlayer deformation is often overlooked [13-15]. In fact, the interlayer vdW interactions for most 2D materials are relatively weak in the mechanical sense, so individual layers with atomically smooth surfaces are highly lubricated [16]. Recently, the interlayer shear behavior has been explored by recording the interaction between multilayer nanosheets and a scanning tip via frictional force microscopy $[17,18]$ or an indentation tip via an atomic force microscope (AFM) $[19,20]$. Although these tip-based measurements have unveiled interesting dynamics due to adhesive and frictional interactions, the understanding of interlayer deformation and failure mechanisms within multilayered 2D structures remains limited, and quantitative characterization of the interlayer interactions has been experimentally challenging and yet to emerge.

Here, we report an experimental measurement of the interlayer shear stress in bilayer graphene and demonstrate 
continuous growth of an interlayer shear zone via a bubble loading device. We uncover that such a process is governed by previously overlooked interlayer shear deformation for the bilayer graphene bubbles, which allows the interlayer shear stress to be determined. To the best of our knowledge, this is the first measurement of the interlayer shear stress between the atomically thin structures which may be extended to understand the interlayer shear interactions in emerging 2D materials and the vdW heterostructures. The capability to probe the interlayer interaction is significant for the assembly and processing of layered 2D materials and further offers a deep insight into the stacking structure-property relations.

To activate interlayer shear deformation in bilayer graphene, we employed uniform pressure via a bulging device as shown in Fig. 1(a), which could also effectively avoid stress concentration that is inevitable in probeactivated shear interactions [20,21]. Such bulging devices, also called blister tests, have been widely used to explore mechanical properties of thin films [22,23], interfacial parameters between films and substrates [15,24], and strain-dependent electronic and photonic properties of 2D crystals $[25,26]$. Figures 1 (b) and 1(c) are the optical and scanning electron microscope (SEM) images of a bilayer graphene specimen. Graphene-sealed microcavities were fabricated by micromechanical cleavage of graphene over patterned holes in a $\mathrm{SiO}_{2}$ substrate. The lateral dimension of an individual graphene sheet was measured by the optical microscopy, and their layer numbers were identified by Raman spectroscopy (Fig. S1 [27]). To create a pressure difference $(\Delta p)$ and control the bulging process, we followed a well-developed gas diffusion method [15] and obtained $\Delta p=\left(p_{0} V_{0} / V_{0}+V_{b}\right)-p_{e}$ according to the ideal gas law, where $p_{0}$ and $p_{e}$ are applied and external atmosphere pressures, $V_{0}$ and $V_{b}$, are volumes of the hole and bubble, respectively. We then utilized $\Delta p$ as a controlling load, pushing the suspended graphene upwards and pulling the substrate-supported graphene (outside the hole) towards the center of the hole by a controllable bulging process. Multiple characterization techniques were employed, including [Fig. 1(a)] in situ Raman spectroscopy to monitor interlayer shear deformation outside the hole
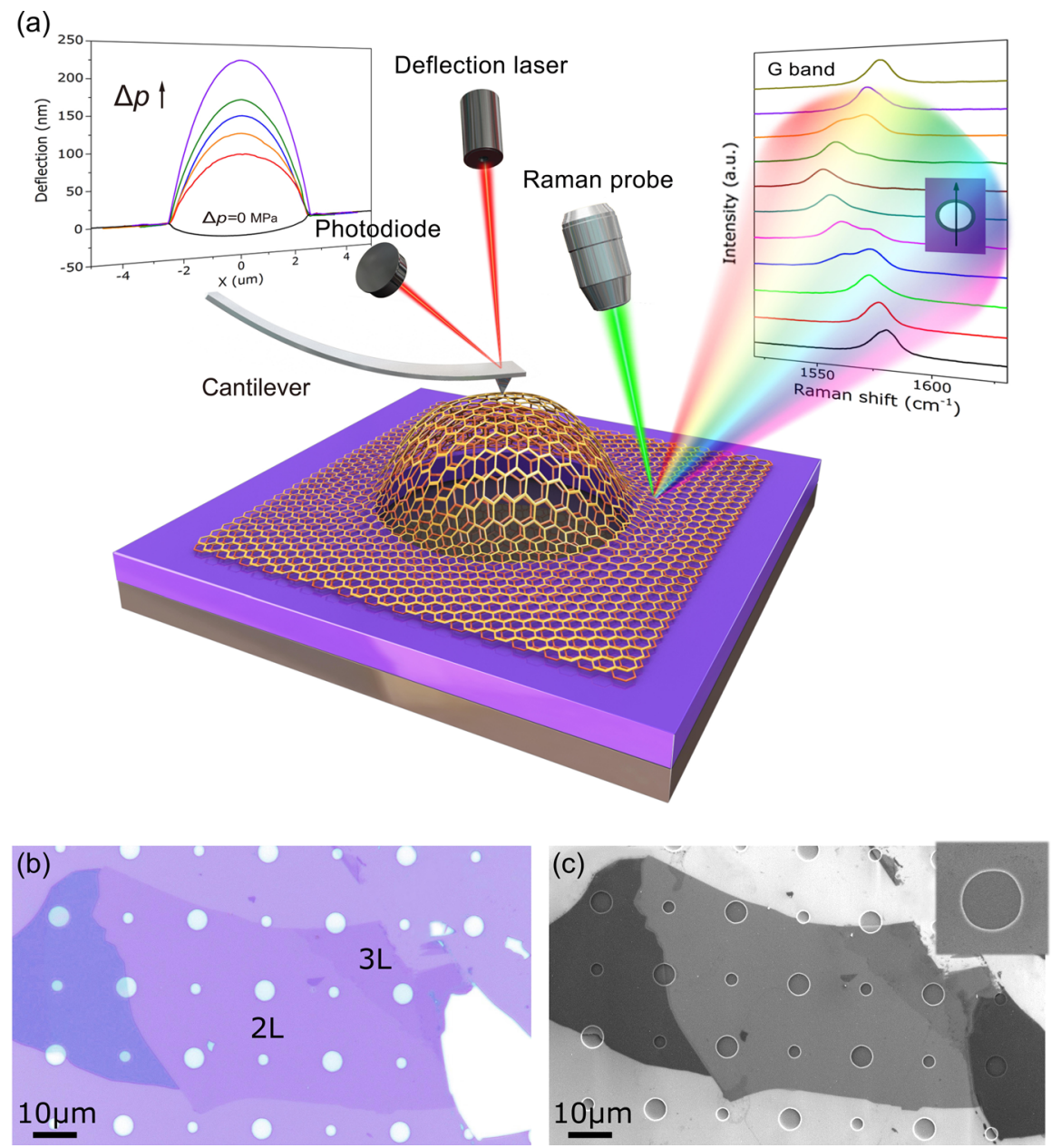

FIG. 1. (a) Schematic diagram of a bilayer graphene bulging device and characterizations. The left inset shows profiles of a graphene bubble with increasing pressures. The right inset shows Raman spectra of a graphene $G$ band stacked vertically in the direction of a line scan. (b) Optical and (c) SEM images of exfoliated graphene sheets on the prepatterned $\mathrm{SiO}_{2}$ substrate. 
and in situ AFM to measure the height profile of each bubble in order to examine the effect of interlayer sliding on the overall bugling behavior. For comparison, monolayer graphene bubbles were also obtained and characterized in this work.

Typical Raman spectra for monolayer and bilayer graphene recorded on the supported regions are shown in Fig. S1 [27]. For monolayer graphene, at $\Delta p=0 \mathrm{MPa}$, the Raman $G$ band is located at $1581 \mathrm{~cm}^{-1}$, which is consistent with previously reported results [40]. In contrast, the suspended region (Fig. S4 [27]) exhibits slight differences in both frequency and FWHM of the $G$ band, which is attributed to the absence of charge transfer doping [40]. With increasing pressure difference, substantial downshifts $\left(\sim 55 \mathrm{~cm}^{-1}\right.$ at $\left.\Delta p=0.35 \mathrm{MPa}\right)$ in the Raman $G$ band are visible in the suspended region of graphene, demonstrating significant tensile strains induced by pressurized bubble formation. Note that, compared with the doping-effectinduced Raman shift, the strain from bubbling is the primary mechanism behind the evolution of the Raman features. As the graphene in the suspended region bulges up, the supported graphene around the hole is pulled towards the center of the hole, and the $G$ band in the supported region shows slight downshifts. For bilayer graphene, a similar downshifting tendency is also observed, both in the supported and the suspended region [27].

We then employ Raman maps plotting the frequency shifts of the $G$ band [41-43], sensitive to strain, to experimentally identify the interlayer (graphene-graphene) and interfacial (graphene- $\mathrm{SiO}_{2}$ ) shear behaviors outside the hole [Figs. 2(a)-2(h)]. Figures 2(c) and 2(f) show the initial Raman $G$-band frequency mappings of as-prepared monolayer and bilayer graphene samples at $p_{0}=p_{e}$, respectively, both exhibiting uniform distributions outside the hole, whereas small frequency shifts are observable at the center of the hole. This indicates a small strain inside the hole and nearly zero strain outside. For monolayer graphene, Figs. 2(c)—2(e) show that the interfacial shear deformation between supported graphene and the $\mathrm{SiO}_{2}$ substrate outside the hole could be continuously activated by the pressure applied in a controllable manner. Unsurprisingly, as the monolayer graphene is bulged upwards with maximum deflection $\delta=155 \mathrm{~nm}$ at the center under $\Delta p=0.14 \mathrm{MPa}$, the $G$ band redshifts significantly inside the hole [Fig. 2(d)]. Meanwhile, the $G$ band of the supported graphene also exhibits redshifting, exhibiting a strain gradient (denoted as the color gradient) in an annular region outside the hole. That is a clear (a)

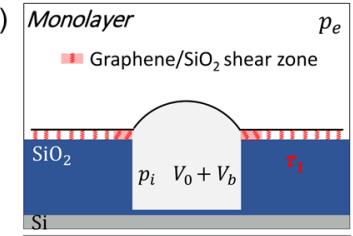

(b)

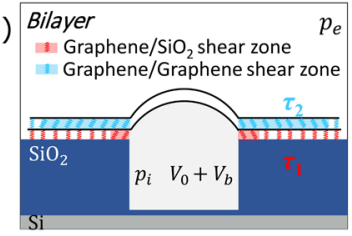

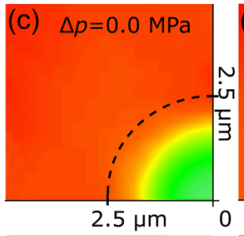
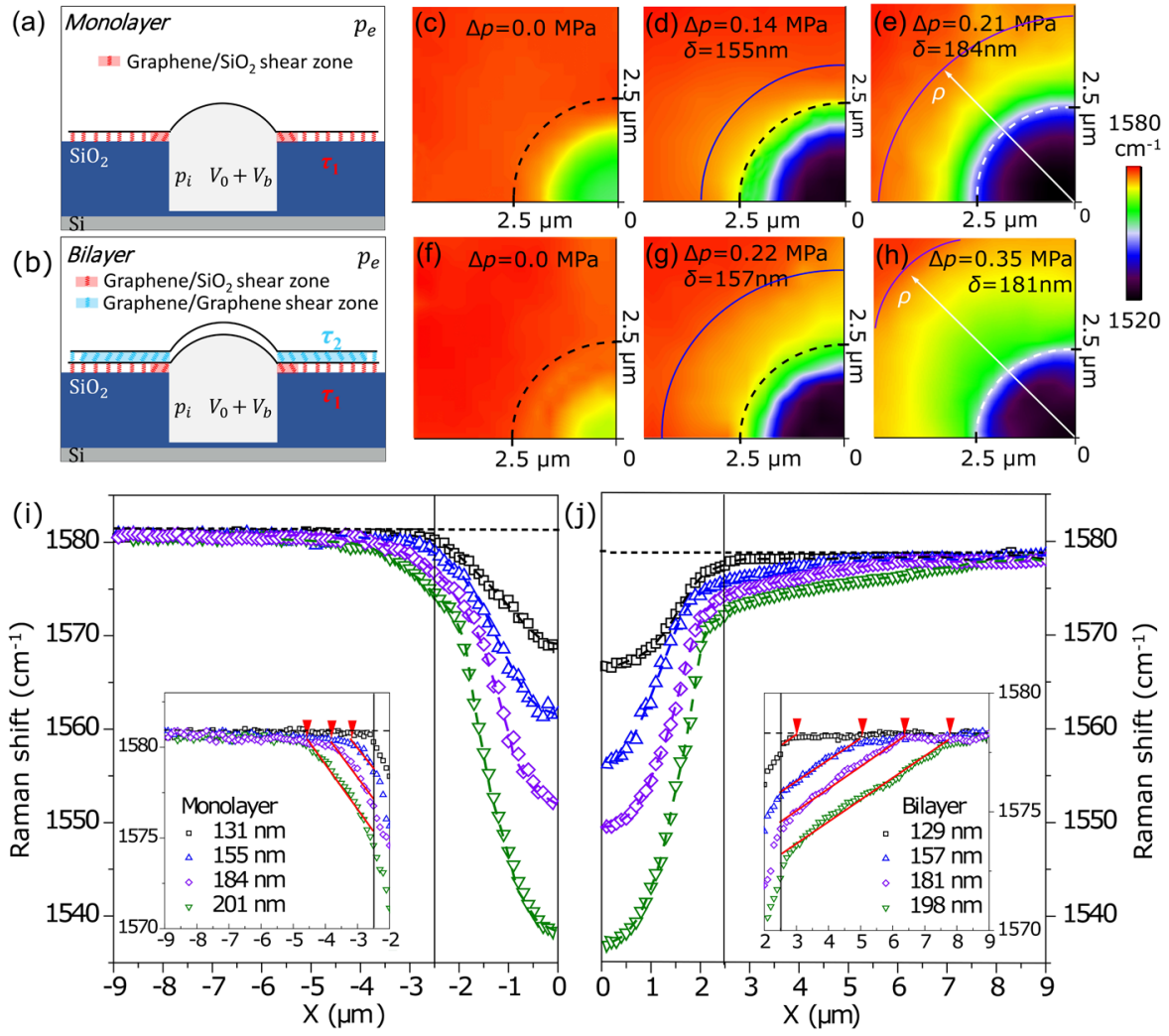

FIG. 2. Schematic diagrams of bulged (a) monolayer and (b) bilayer graphene samples. Raman contour maps of $G$-band frequency in one quadrant revealed the strain distributions in (c) - (e) monolayer and (f) - (h) bilayer graphene samples in both suspended and supported regions at different pressures. The cross sections of Raman contour maps are shown for (i) monolayer and (j) bilayer graphene. The insets show the variation of the $G$-band frequency near the edges of the hole. Solid vertical lines are positioned at the edges of the hole, and the dashed horizontal lines indicate the frequency of the $G$ band at zero strain. 
indication of interfacial shear deformation of the supported graphene. As illustrated in Fig. 2(a), the applied pressure inside the hole pushes the graphene upwards and induces a tension at the edge of the hole, pulling the supported graphene towards the hole. On the other hand, an interfacial shear stress, $\tau_{1}$, between graphene and the substrate opposes interfacial sliding. The competition leads to a finite outer radius for the annular shear zone, beyond which the supported graphene remains strain free. Within the shear zone, Raman redshift or the corresponding strain level decreases with the distance from the edge of the hole until the $G$ band returns to the unstrained level. We define a dimensionless parameter $\rho$ as the ratio between the outer radius of the shear zone (the solid line) and the radius of the hole (the dashed line). As the applied pressure increases, the shear zone expands with an increasing outer radius. At $\Delta p=0.21 \mathrm{MPa}$, the Raman mapping [Fig. 2(e)] shows larger redshifts in both suspended and supported regions. Moreover, the shear zone in the supported region grows controllably outwards with a larger $\rho$, implying that more interfacial area outside the hole participated in the shear deformation to balance the increased pressure inside the hole.

For bilayer graphene samples, similar growth behavior of a shear zone outside the hole could also be observed from Raman mappings [Figs. 2(f)—2(h)]. Compared to monolayer graphene, bilayer graphene requires a larger pressure difference to bulge up to the same maximum deflection, yielding a similar strain distribution inside the hole but a much larger shear zone outside the hole. These results suggest that for bilayer graphene bubbles, significant interlayer shear deformation occurred outside the hole. As illustrated in Fig. 2(b), the interlayer shear zone would be much larger than the interfacial shear zone for the monolayer graphene if the interlayer shear stress $\tau_{2}$ between the two graphene layers is much smaller than $\tau_{1}$ [44]. In other words, the much more discernable growth behavior or the larger shear zone observed in bilayer samples outside the hole is dominated by the shear deformation at the weak graphenegraphene interface rather than the graphene-substrate interface, which also strongly agrees with our Raman mapping results of the $G$-peak full width at half maximum (FWHM) $[27,45]$. Note that, in previous works [15,46], pressurized blister tests were commonly performed to measure the adhesion energy between thin films and substrates as interfaces would delaminate at a critical pressure. Here, however, the interlayer shear deformation could be actuated under relatively low pressures (far from that required to cause delamination [27]), due to the weak interlayer shear resistances.

To determine the nature of the interlayer shear zone outside the hole, we further controlled the growth of the shear zone continuously by tuning the applied pressure and recorded line-scanning frequency shifts of the Raman $G$ band [Figs. 2(i) and 2(j)]. Details in the insets clearly show the Raman shift as a function of the spatial position in the monolayer and bilayer graphene samples. Interestingly, the measured Raman shift exhibited nearly linear characteristics near the edge of the shear zone, typically implying a constant strain gradient due to a constant interfacial shear stress in one-dimensional scenarios [47,48]. Such linearity also enabled us to clearly determine the radius ratio $\rho$ for the annular shear zones (marked by red lines and symbols), with respect to $\Delta p$. In principle, the biaxial strain and stress in graphene may also be determined from the measured Raman shifts for both monolayer and bilayer graphene samples, which could then be used to determine the shear stresses for the graphene- $\mathrm{SiO}_{2}\left(\tau_{1}\right)$ and graphene-graphene $\left(\tau_{2}\right)$ interface. However, a precise relationship between the Raman shift and the biaxial strain of graphene (e.g., the Grüneisen parameter and shear deformation potential) is still challenging to establish when the interlayer coupling exists for bilayer graphene [49]. This issue might be resolved if independent Raman signals could be obtained for both bottom and top layers [50]. Here, we determine the interlayer shear stress based on the measurement of the shear zone size $(\rho)$ from Raman mapping, which does not require precise strain measurements.

Previous works typically focused on the overall mechanical performance of multilayer graphene sheets and thus simplified them as a single layer with an in-plane stiffness of $n E t$, where $n$ is the number of layers [15,51]. However, since the interlayer shear resistance is fairly weak, we analyze the deformation of the bilayer graphene as two monolayers with different interfacial shear stresses for graphene- $\mathrm{SiO}_{2}\left(\tau_{1}\right)$ and graphene-graphene $\left(\tau_{2}\right)$ interfaces in supported regions [27]. Of particular interest is the fact that the growth of the shear zone for both the monolayer and bilayer graphene bubbles could be well described by the analytical model, which allows determination of $\tau_{1}$ and $\tau_{2}$ based on measurements of $\delta$ and $\rho$.

As Raman shifts cannot provide direct evidence of the interlayer deformations at atomic-scale resolution, where the mechanism of interlayer load transfer through shear could be revealed, we carried out molecular dynamics (MD) simulations for the bulging tests. The results depicted in Figs. 3(a) and 3(b) clearly demonstrate the binding energy and radial displacement of a bilayer graphene sheet, indicating that there is no debonding in our MD simulations in consistent with the experiments. The interlayer shear deformation can be visualized from the relative atomic displacements between top-layer and bottom-layer graphene as shown in Figs. 3(c) and 3(d). Similar to the Raman observation in Fig. 2, a clear zone (undergoing a strain gradient) outside the hole could be directly observed by simulations (Fig. S21 [27]). In addition, we find that the strain distributions in both the suspended and supported regions by MD simulations can be well fitted to our analytical methods, validating our theoretical model for analyzing the experimental results. 
(a)

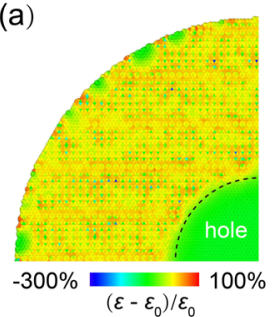

(b)

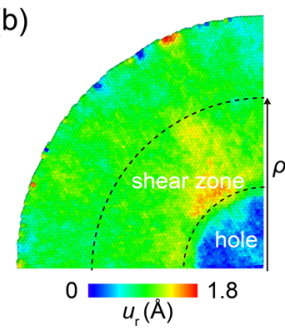

(c)

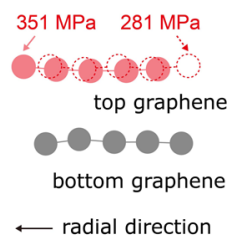

(d)

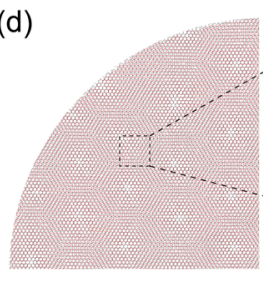

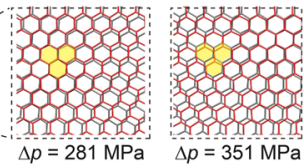

FIG. 3. (a) Spatial distribution of the interfacial binding energy between bottom-layer graphene and the silica substrate. (b) Decomposed in-plane displacement $u_{r}$ in the top-layer graphene calculated from the MD simulation results. (c,d) The interlayer shear behaviors of bottom-layer and top-layer graphene, resolved by the atomic displacement under specific pressure difference.

We could determine the interfacial and interlayer shear stresses based on the measurements of $\delta$ and $\rho$ with respect to $\Delta p$ in bulging tests of monolayer and bilayer graphene. In Fig. 4(a), we plot $\rho$ versus $\Delta p$ for monolayer graphene bubbles. Following the steps outlined in Fig. S12 [27], $\tau_{1}$ is extracted with values in the range of $1-3 \mathrm{MPa}$ and an average of 1.64 MPa (Fig. 5), in good agreement with previous reports for the graphene- $\mathrm{SiO}_{2}$ interfaces (0.25-2 MPa) [51,52] and graphene-polymer interfaces $(0.3-2.3 \mathrm{MPa})[43,53]$. In contrast, for bilayer graphene,
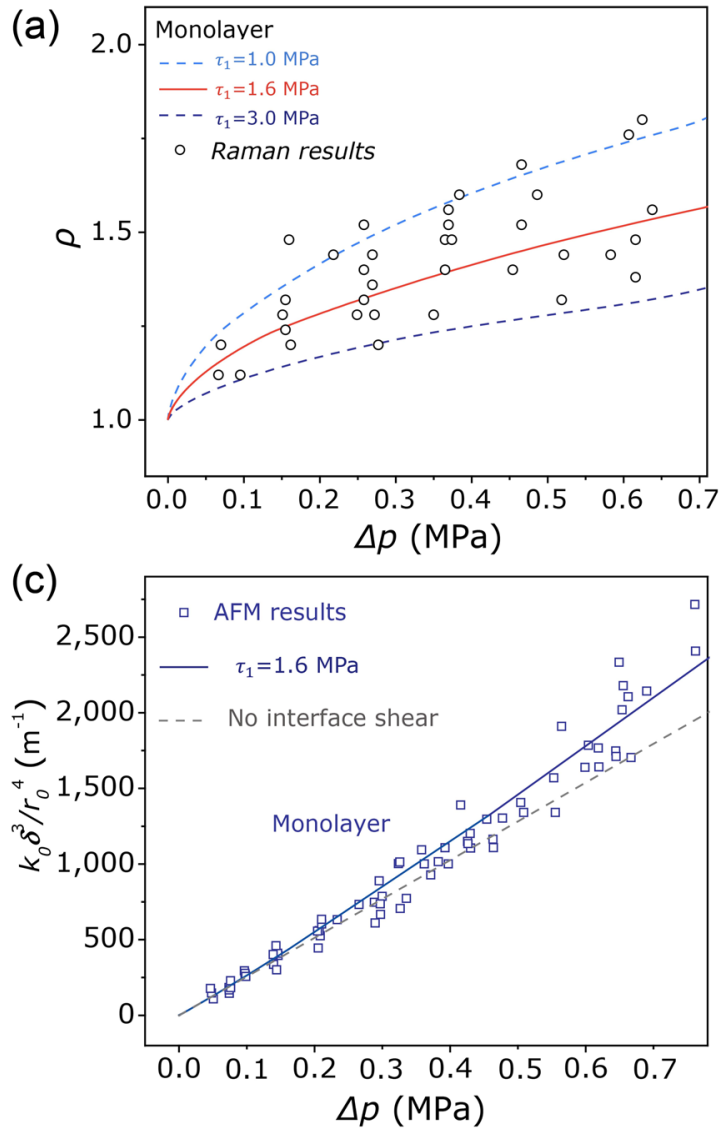

the shear deformation outside the hole is much more significant, owing to the weak shear resistance between the two graphene layers. As shown in Fig. 4(b), the observed growth behavior of the interlayer shear zone is governed by an $\tau_{2}$ of $0.02-0.06 \mathrm{MPa}$ with an average of $0.04 \mathrm{MPa}$ (Fig. 5), 2 orders of magnitude lower than that of the graphene-substrate interface. Similar shear stresses have been experimentally obtained for multi-walled carbon nanotubes (MWCNTs) and graphite, demonstrating the ultralow interlayer shear interactions dominated by vdW forces [54].

(b)

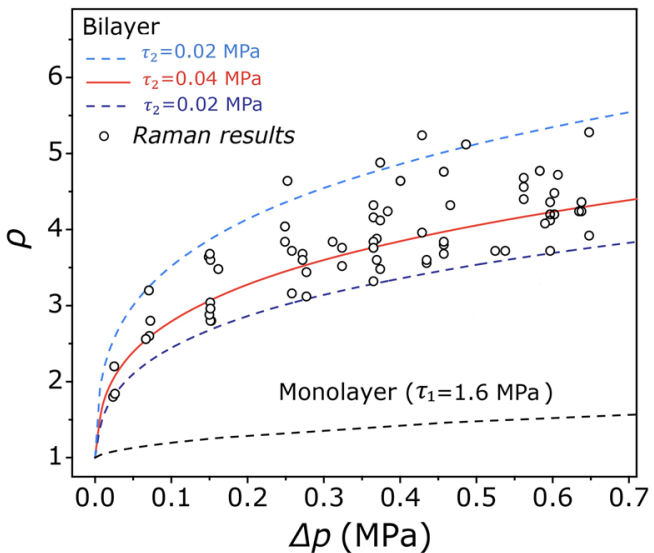

(d)

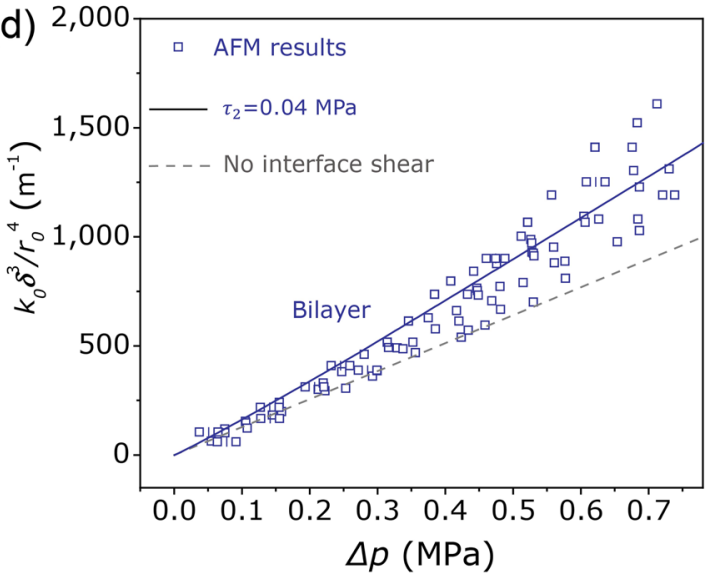

FIG. 4. The growth of the (a) interfacial and (b) interlayer shear zone versus $\Delta p$ for monolayer and bilayer graphene bubbles. The lines are predicted by the membrane analysis with different interfacial shear stresses. Normalized center deflection versus $\Delta p$ for (c) monolayer and (d) bilayer graphene bubbles. Solid lines are predicted by the membrane analysis with Et $=390 \mathrm{~N} / \mathrm{m}$ and $\tau_{1}=1.64 \mathrm{MPa}$ for monolayer and $\tau_{2}=0.04 \mathrm{MPa}$ for bilayer. Dashed lines are predictions without considering interfacial or interlayer shear deformations in the supported regions. 


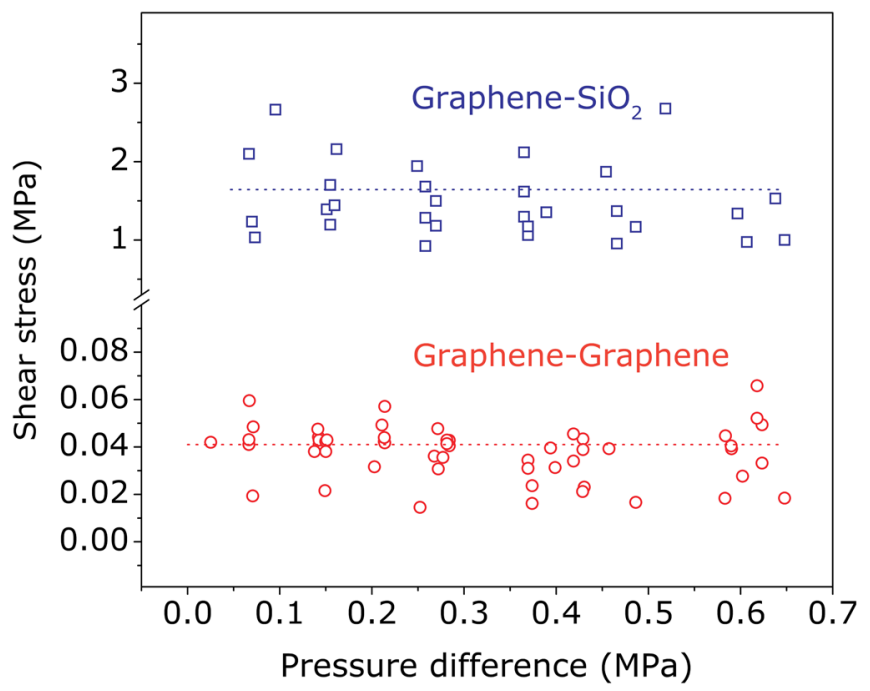

FIG. 5. Measured shear stresses for graphene- $\mathrm{SiO}_{2}$ and graphene-graphene interfaces. Dashed lines correspond to the average values of $1.64 \mathrm{MPa}$ and $0.04 \mathrm{MPa}$.

Some modeling works and bending experiments have reported considerably high shear stress between graphene layers $(\sim 0.1 \mathrm{GPa})$ due to the commensurability-dependent stick-slip friction with periodically changing peak shear stress $[55,56]$. Instead, the relatively lower values here can be attributed to the averaged effect caused by the limited spatial resolution of the Raman laser spot $(\sim 1 \mu \mathrm{m})$.

We further discuss the effects of the interfacial and interlayer shear on the out-of-plane deflection of graphene bubbles based AFM characterizations. The membrane analysis [27] predicts a relation between $\Delta p$ and $\delta$ at the center of the hole as

$$
\Delta p=k E t \frac{\delta^{3}}{r_{0}^{4}},
$$

where $E t$ is the in-plane stiffness for monolayer graphene, $r_{0}$ is the radius of the hole, and $k$ is a dimensionless coefficient that depends on the pressure difference and the interfacial shear stress. Previous works have typically ignored the shear deformation in the supported region outside the hole [15], leading to a constant $k$ (defined as $k_{0}=3.10$ for graphene) and hence a linear relation between $\delta^{3}$ and $\Delta p$, with a slope proportional to the inplane stiffness of graphene, Et. However, the presence of shear deformation outside the hole can result in a nonlinear behavior as $k$ decreases with increasing pressure (Fig. S9 [27]), which is observed in Fig. 4(c) plotting $k_{0}\left(\delta^{3} / r_{0}^{4}\right)$ versus $\Delta p$ for monolayer graphene bubbles. In comparison with the prediction assuming no shear deformation outside the hole, the prediction with a finite shear stress $\left(\tau_{1}=\right.$ $1.64 \mathrm{MPa}$ ) offers a better agreement with the AFM measurements, especially at relatively high pressure levels where more significant shear deformation occurred.
Compared to monolayer graphene bubbles, more significant shear deformation occurs between two graphene layers for bilayer graphene bubbles. Consequently, the bilayer graphene bubbles exhibited a markedly nonlinear relationship between $k_{0}\left(\delta^{3} / r_{0}^{4}\right)$ and $\Delta p$ [Fig. 4(d)]. Similar behaviors could also be observed in a previous work on few-layer graphene sheets [15]. Thus, the effects of the weak interlayer shear resistance $\left(\tau_{2}=0.04 \mathrm{MPa}\right)$ should be considered in efforts to predict the overall bulging deformation of bilayer and few-layer graphene sheets. As illustrated in Fig. S14 [27], a bilayer graphene bubble may be treated as a superposition of two monolayer graphene bubbles, each subjected to a pressure difference in the suspended region and an interfacial shear stress in the supported region. Using Eq. (1) for each monolayer, the total pressure difference for the bilayer is related to the center deflection [Fig. 4(d) solid line] as $\Delta p=\left(k_{1}+k_{2}\right) E t\left(\delta^{3} / r_{0}^{4}\right)$, where $k_{1}$ and $k_{2}$ depend on two shear stresses $\left(\tau_{1}\right.$ and $\tau_{2}$ ) for bottom and top layers, respectively. Furthermore, the interlayer shear zones in bilayer graphene bubbles could grow to reach the outer boundary of graphene sheets, leading to slightly reduced stiffness as predicted by the membrane analysis (Fig. S18) and MD simulations (Fig. S22) [27].

In conclusion, we have observed controllable growth of the interlayer shear zone in the supported region of bilayer graphene bubbles using the pressurized bulging devices. By combining Raman and AFM measurements with a membrane analysis, we have demonstrated a method for determining the interfacial and interlayer shear stresses for the bilayer graphene as one of the thinnest structures possible. This is the first measurement of the interlayer shear stress for graphene. This result can be used to guide developments of graphene-based electrical and mechanical devices where interlayer shear is known to play a fundamentally important role. Our results could also open up ample opportunities for fundamental studies on interfacial and interlayer deformations in 2D heterostructures consisting of various 2D materials in the multilayer form.

This project was jointly supported by the National Key Basic Research Program of China (Grant No. 2013CB934203), the National Key Research and Development Program of China (Grant No. 2016YFA0301204) and the National Natural Science Foundation of China (Grants No. 21474023, No. 11225210, No. 11434010, No. 11474277 and No. 11225421).

G. W. and Z. D. contributed equally to this work.

\footnotetext{
*To whom all correspondence should be addressed. liulq@nanoctr.cn

${ }^{\dagger}$ To whom all correspondence should be addressed. xuzp@tsinghua.edu.cn

*To whom all correspondence should be addressed. zhong.zhang@ nanoctr.cn.
} 
[1] A. K. Geim and K. S. Novoselov, Nat. Mater. 6, 183 (2007).

[2] X. S. Li et al., Science 324, 1312 (2009).

[3] K. S. Novoselov, A. K. Geim, S. Morozov, D. Jiang, Y. Zhang, S. Dubonos, I. Grigorieva, and A. Firsov, Science 306, 666 (2004).

[4] J. S. Bunch, A. M. van der Zande, S. S. Verbridge, I. W. Frank, D. M. Tanenbaum, J. M. Parpia, H. G. Craighead, and P. L. McEuen, Science 315, 490 (2007).

[5] K. M. F. Shahil and A. A. Balandin, Nano Lett. 12, 861 (2012).

[6] R. R. Nair, H. A. Wu, P. N. Jayaram, I. V. Grigorieva, and A. K. Geim, Science 335, 442 (2012).

[7] Z. Dai, Y. Wang, L. Liu, X. Liu, P. Tan, Z. Xu, J. Kuang, Q. Liu, J. Lou, and Z. Zhang, Adv. Funct. Mater. 26, 7003 (2016).

[8] A. K. Geim and I. V. Grigorieva, Nature (London) 499, 419 (2013).

[9] J. W. Hutchinson and Z. Suo, Adv. Appl. Mech. 29, 63 (1992).

[10] Y. Gao et al., Nat. Mater. 14, 714 (2015).

[11] P. H. Tan et al., Nat. Mater. 11, 294 (2012).

[12] K. S. Novoselov, A. Mishchenko, A. Carvalho, and A. H. C. Neto, Science 353, aac9439 (2016).

[13] S. Bertolazzi, J. Brivio, and A. Kis, ACS Nano 5, 9703 (2011).

[14] H. Conley, N. V. Lavrik, D. Prasai, and K. I. Bolotin, Nano Lett. 11, 4748 (2011).

[15] S. P. Koenig, N. G. Boddeti, M. L. Dunn, and J. S. Bunch, Nat. Nanotechnol. 6, 543 (2011).

[16] Z. Liu, J. Yang, F. Grey, J. Z. Liu, Y. Liu, Y. Wang, Y. Yang, Y. Cheng, and Q. Zheng, Phys. Rev. Lett. 108, 205503 (2012).

[17] Z. Deng, A. Smolyanitsky, Q. Y. Li, X. Q. Feng, and R. J. Cannara, Nat. Mater. 11, 1032 (2012).

[18] T. Filleter, J. L. McChesney, A. Bostwick, E. Rotenberg, K. V. Emtsev, T. Seyller, K. Horn, and R. Bennewitz, Phys. Rev. Lett. 102, 086102 (2009).

[19] A. Falin et al., Nat. Commun. 8, 15815 (2017).

[20] X. Wei, Z. Meng, L. Ruiz, W. Xia, C. Lee, J. W. Kysar, J. C. Hone, S. Keten, and H. D. Espinosa, ACS Nano 10, 1820 (2016).

[21] C. Lee, Q. Li, W. Kalb, X. Z. Liu, H. Berger, R. W. Carpick, and J. Hone, Science 328, 76 (2010).

[22] J. U. Lee, D. Yoon, and H. Cheong, Nano Lett. 12, 4444 (2012).

[23] J. J. Vlassak and W. D. Nix, J. Mater. Res. 7, 3242 (1992).

[24] Z. Cao, P. Wang, W. Gao, L. Tao, J. W. Suk, R. S. Ruoff, D. Akinwande, R. Huang, and K. M. Liechti, Carbon 69, 390 (2014).

[25] D. Metten, F. Federspiel, M. Romeo, and S. Berciaud, Phys. Rev. Applied 2, 054008 (2014).

[26] J. Zabel, R. R. Nair, A. Ott, T. Georgiou, A. K. Geim, K. S. Novoselov, and C. Casiraghi, Nano Lett. 12, 617 (2012).

[27] See Supplementary Material at http://link.aps.org/ supplemental/10.1103/PhysRevLett.119.036101, which includes Refs. [28-39], for experimental setup and characterizations, analysis based on membrane mechanics, and molecular simulations.
[28] J. Canet-Ferrer, E. Coronado, A. Forment-Aliaga, and E. Pinilla-Cienfuegos, Nanotechnology 25, 395703 (2014).

[29] C. Metzger, S. Rémi, M. Liu, S. V. Kusminskiy, A. H. Castro Neto, A. K. Swan, and B. B. Goldberg, Nano Lett. 10, 6 (2010).

[30] P. Wang, W. Gao, Z. Cao, K. M. Liechti, and R. Huang, J. Appl. Mech. 80, 040905 (2013).

[31] S. Timoshenko and J. M. Gere, Theory of Elastic Stability (Dover Publications, New York, 2012).

[32] H. Hencky, Z. Math. Phys. 63, 311 (1915).

[33] L. Landau, E. Lifshitz, A. Kosevich, and L. Pitaevskii, Theory of Elasticity (Pergamon Press, Oxford, 1986).

[34] W. Fichter, NASA Technical Paper No. 3658 (1997).

[35] T. Zhang, X. Li, and H. Gao, Int. J. Fract. 196, 1 (2015).

[36] S. Plimpton, J. Comput. Phys. 117, 1 (1995).

[37] B. Ni, K. H. Lee, and S. B. Sinnott, J. Phys. Condens. Matter 16, 7261 (2004).

[38] S. Munetoh, T. Motooka, K. Moriguchi, and A. Shintani, Comput. Mater. Sci. 39, 334 (2007).

[39] F. Shimizu, S. Ogata, and J. Li, Mater. Trans., JIM 48, 2923 (2007).

[40] S. Berciaud, S. Ryu, L. E. Brus, and T. F. Heinz, Nano Lett. 9, 346 (2009).

[41] A. C. Ferrari and D. M. Basko, Nat. Nanotechnol. 8, 235 (2013).

[42] O. Frank, G. Tsoukleri, I. Riaz, K. Papagelis, J. Parthenios, A. C. Ferrari, A. K. Geim, K. S. Novoselov, and C. Galiotis, Nat. Commun. 2, 255 (2011).

[43] R. J. Young, I. A. Kinloch, L. Gong, and K. S. Novoselov, Compos. Sci. Technol. 72, 1459 (2012).

[44] Z. J. Wang, J. Dong, Y. Cui, G. Eres, O. Timpe, Q. Fu, F. Ding, R. Schloegl, and M. G. Willinger, Nat. Commun. 7, 13256 (2016).

[45] C. Neumann et al., Nat. Commun. 6, 8429 (2015).

[46] K.-T. Wan and Y.-W. Mai, Acta Metall. Mater. 43, 4109 (1995).

[47] Z. Dai, G. Wang, L. Liu, Y. Hou, Y. Wei, and Z. Zhang, Compos. Sci. Technol. 136, 1 (2016).

[48] G. Wang, Z. Dai, L. Liu, H. Hu, Q. Dai, and Z. Zhang, ACS Appl. Mater. Interfaces 8, 22554 (2016).

[49] M. Huang, H. Yan, C. Chen, D. Song, T. F. Heinz, and J. Hone, Proc. Natl. Acad. Sci. U.S.A. 106, 7304 (2009).

[50] Y. He et al., Nano Lett. 16, 3314 (2016).

[51] A. L. Kitt, Z. Qi, S. Remi, H. S. Park, A. K. Swan, and B. B. Goldberg, Nano Lett. 13, 2605 (2013).

[52] X. Wang, K. Tantiwanichapan, J. W. Christopher, R. Paiella, and A. K. Swan, Nano Lett. 15, 5969 (2015).

[53] L. Gong, I. A. Kinloch, R. J. Young, I. Riaz, R. Jalil, and K. S. Novoselov, Adv. Mater. 22, 2694 (2010).

[54] A. Kis, K. Jensen, S. Aloni, W. Mickelson, and A. Zettl, Phys. Rev. Lett. 97, 025501 (2006).

[55] L. Ruiz, W. Xia, Z. Meng, and S. Keten, Carbon 82, 103 (2015).

[56] X. Chen, C. Yi, and C. Ke, Appl. Phys. Lett. 106, 101907 (2015). 